\title{
Optical real-time biopsy by endocytoscopy: a case of sessile serrated lesion with dysplasia
}

Sessile serrated lesions (SSLs) are early precursor lesions in the serrated neoplasia pathway, which progresses through SSL with dysplasia (SSLD) to invasive carcinoma. In SSLD, certain endoscopic characteristics, including double elevation, central depression, and reddishness, have been found frequently, and type IIIL, IV, and VI pit patterns were observed more frequently using magnifying chromoendoscopy with indigo carmine or crystal violet staining [1]. Endocytoscopy is useful for distinguishing SSLD [2], but the endocytoscopy findings of SSLD are poorly understood. Herein, we report a case of SSLD observed using endocytoscopy ( $\triangleright$ Video 1$)$.

A 71-year-old man underwent a colonoscopy, which revealed an area of slightly elevated mucosa in the cecum, with reddish nodules on the oral and anal sides of the lesion ( $\triangleright$ Fig. $1 \mathbf{a}$ ). Magnifying narrow-band imaging (NBI) showed no vessel pattern in the slightly elevated area, and dilated and branching vessels were seen in the reddish nodules ( $\mathbf{F i g . 1} \mathbf{b}$ and $>$ Fig. $\mathbf{2}$ a-c). With chromoendoscopy with indigo carmine, the boundary of the lesion was clearly visible ( Fig. 1 c). Magnifying chromoendoscopy using crystal violet staining showed a type II open pit pattern in the slightly elevated central area and the reddish nodule on the oral side of the lesion ( $\mathbf{F i g . 2 d , e ) . ~ T y p e ~ I I I L ~ p i t s ~ w e r e ~}$ seen in the reddish nodule on the anal side of the lesion ( $\triangleright$ Fig.2f). Endocytoscopy using $1.0 \%$ methylene blue and $0.05 \%$ crystal violet showed oval lumens and small round nuclei in the slightly elevated area and in the reddish nodule on the anal side of the lesion ( $\mathbf{F i g .} \mathbf{3} \mathbf{b}$, c). Irregular lumens and swollen roundish nuclei were seen in the nodule on the oral side ( $\triangleright$ Fig. $\mathbf{3}$ a; $\triangleright$ Video 1 ). Therefore, we diagnosed SSLD and en bloc endoscopic resection was performed. Histopathological findings revealed an SSLD in the reddish nodule that was endoscopically on the oral side of the lesion ( $\vee$ Fig. 4 and Fig. 5)

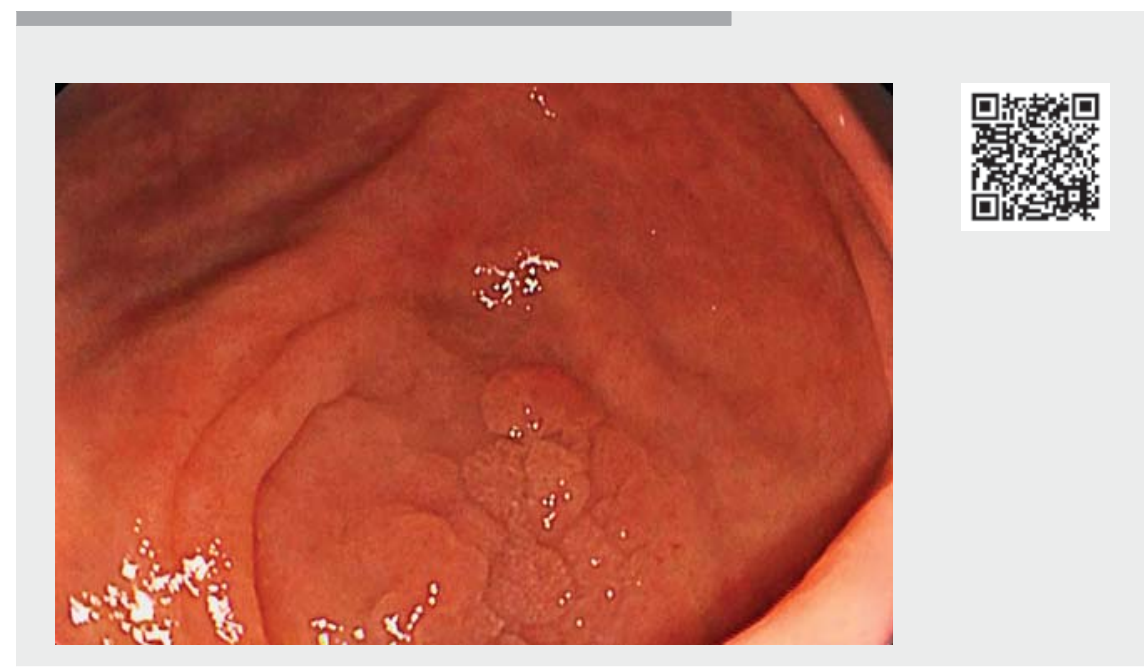

Video 1 A sessile serrated lesion with dysplasia found in the cecum is observed by endocytoscopy.
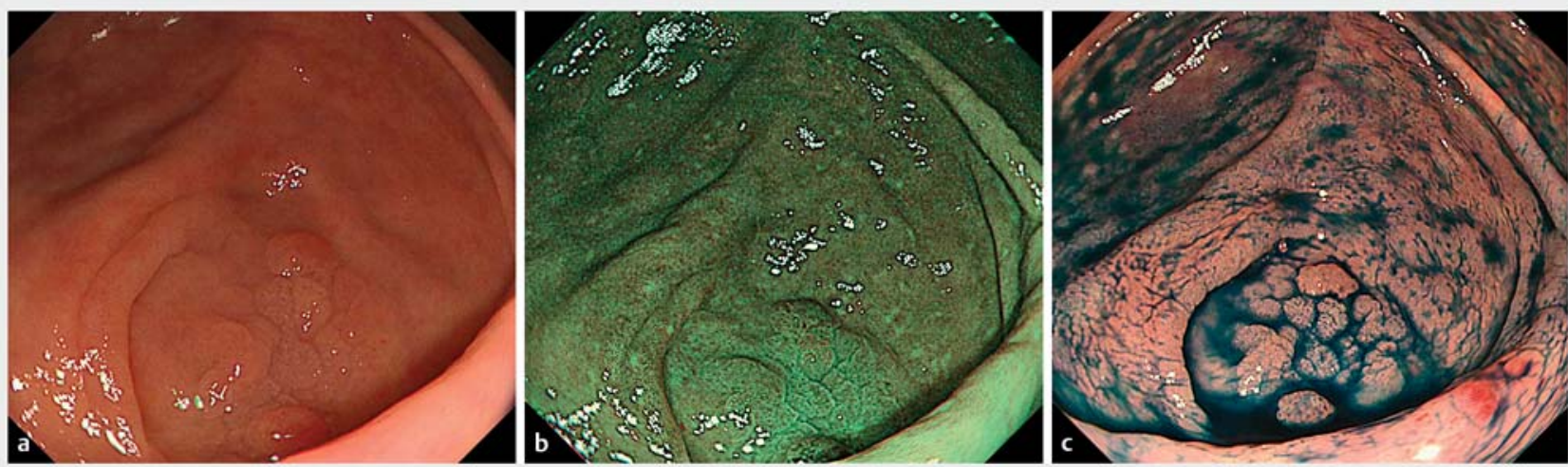

- Fig. 1 Endoscopic images showing a slightly elevated area of mucosa in the cecum: $\mathbf{a}$ in white light; $\mathbf{b}$ on narrow-band imaging; $\mathbf{c}$ on chromoendoscopy using indigo carmine. 

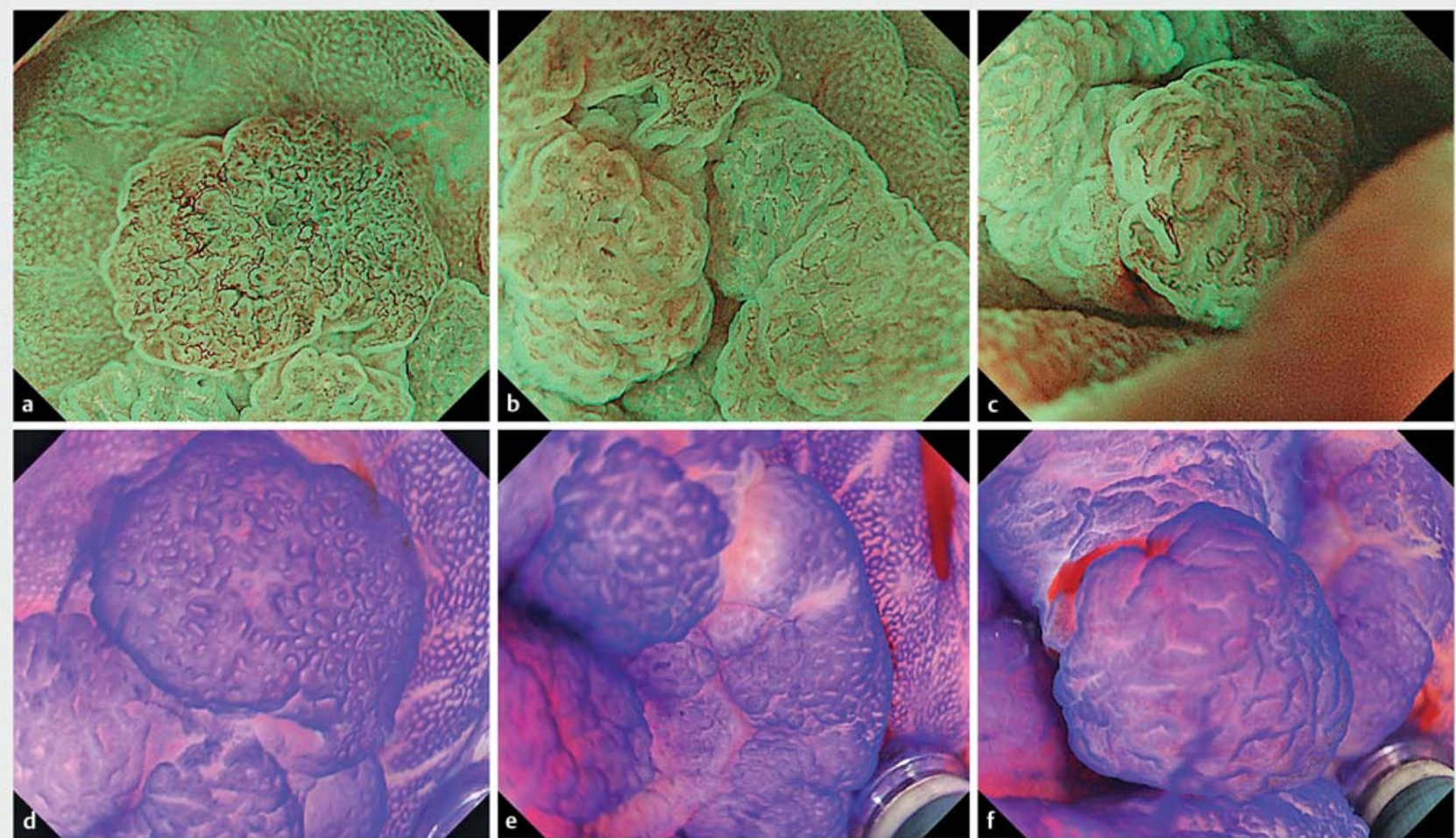

- Fig. 2 Magnifying images with: a-c narrow-band imaging; d-f chromoendoscopy using crystal violet staining showing: a dilated and branching vessels in the reddish nodule on the oral side of the lesion; $\mathbf{b}$ no vessel pattern in the central slightly elevated area; $\mathbf{c}$ dilated and branching vessels in the reddish nodule on the anal side of the lesion; $\mathbf{d}$ type II open pit pattern in the nodule on the oral side; $\mathbf{e}$ type II open pit pattern in the slightly elevated lesion; $\mathbf{f}$ type IIIL pit pattern in the nodule on the anal side.
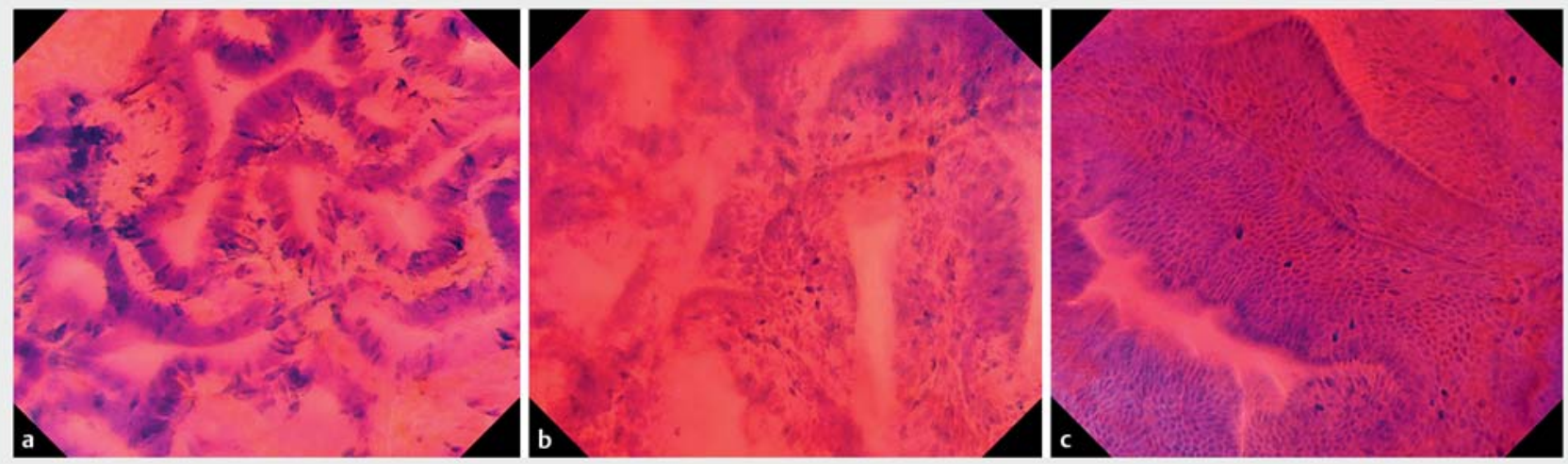

Fig. 3 Endocytoscopy images showing: a irregular lumens and swollen roundish nuclei in the reddish nodule on the oral side of the lesion; b oval lumens and small round nuclei in the central slightly elevated lesion; c oval lumens and small round nuclei in the reddish nodule on the anal side of the lesion.

In this case, two reddish nodules were observed. Magnifying chromoendoscopy showed suspected dysplasia in the reddish nodule on the anal side of the lesion, but endocytoscopy was correctly able to identify the dysplasia in the nodule on the oral side of the lesion from the findings of irregular lumens and swollen roundish nuclei.

Endoscopy_UCTN_Code_TTT_1AQ_2AD

\section{Competing interests}

The authors declare that they have no conflict of interest. 


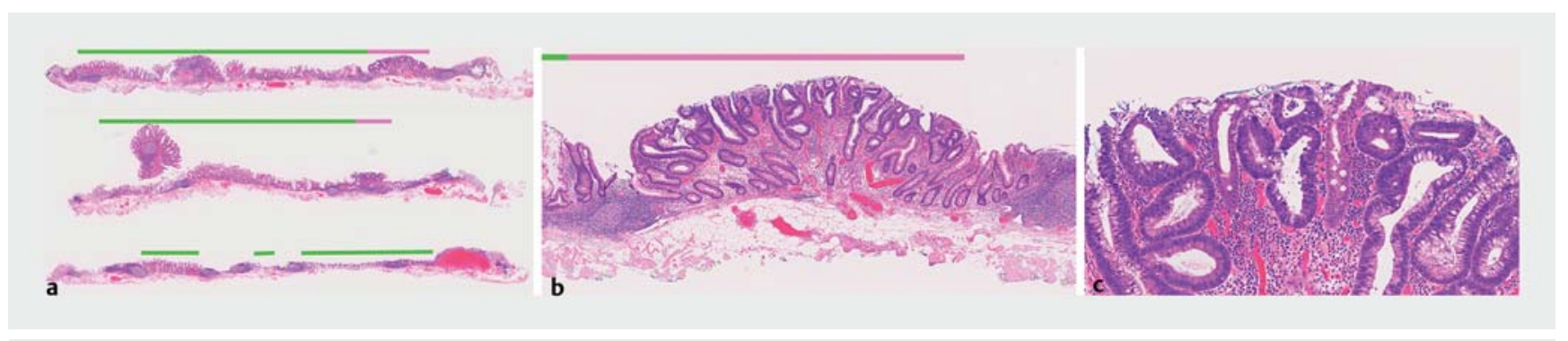

- Fig. 4 Histological examination (hematoxylin and eosin stained) showing: a the distribution of the lesion in the resected specimen, with the green lines indicating areas with sessile serrated lesion and the pink lines areas with dysplasia; $\mathbf{b}$ the area of dysplasia; $\mathbf{c}$ a magnified view of the area of dysplasia, with visible tumor cells being columnar and having large hyperchromatic nuclei.
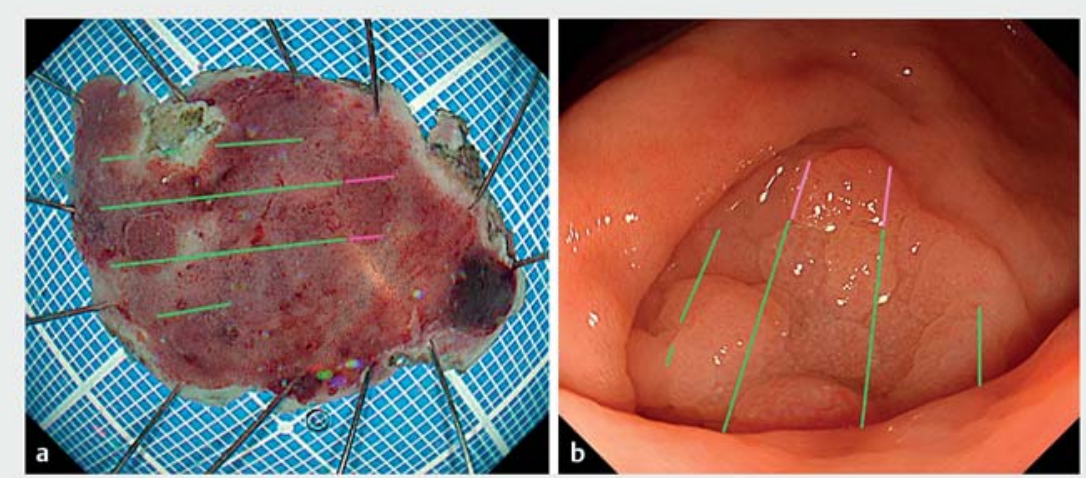

- Fig. 5 Distribution of the lesion: a within the resected specimen on macroscopic view; b on the endoscopic white light view prior to resection.

The authors

Kengo Kasuga', Yutaka Saito', Hiroyuki Takamaru' ${ }^{1}$, Masayoshi Yamada ${ }^{1}$ ๑, Taku Sakamoto', Shigeki Sekine², Toshio Uraoka ${ }^{3}$ 우

1 Endoscopy Division, National Cancer Center Hospital, Tokyo, Japan

2 Pathology Division, National Cancer Center Hospital, Tokyo, Japan

3 Department of Gastroenterology and Hepatology, Gunma University Graduate School of Medicine, Maebashi, Japan

\section{Corresponding author}

\section{Yutaka Saito, MD, PhD}

Endoscopy Division, National Cancer Center Hospital, 5-1-1 Tsukiji, Chuo-ku, Tokyo 104-0045, Japan

ytsaito@ncc.go.jp

\section{References}

[1] Murakami T, Sakamoto N, Ritsuno H et al. Distinct endoscopic characteristics of sessile serrated adenoma/polyp with and without dysplasia/carcinoma. Gastrointest Endosc 2017; 85: 590-600

[2] Kutsukawa M, Kudo S-E, Ikehara $\mathrm{N}$ et al. Efficiency of endocytoscopy in differentiating types of serrated polyps. Gastroint Endosc 2014; 79: 648-656

\section{Bibliography}

Endoscopy 2022; 54: E249-E251

DOI 10.1055/a-1443-4276

ISSN 0013-726X

published online 18.6.2021

(c) 2021. Thieme. All rights reserved.

Georg Thieme Verlag KG, Rüdigerstraße 14, 70469 Stuttgart, Germany

\section{ENDOSCOPY E-VIDEOS}

https://eref.thieme.de/e-videos

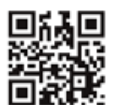

Endoscopy E-Videos is an open access online section, reporting on interesting cases and new techniques in gastroenterological endoscopy. All papers include a high quality video and all contributions are freely accessible online. Processing charges apply (currently EUR 375), discounts and wavers acc. to HINARI are available.

This section has its own submission website at

https://mc.manuscriptcentral.com/e-videos 